maldehyde, hydrogen peroxide, phenol, boric, gallic, hydrochloric and sulfuric acids, sodium borate and sodium benzoate.

(4) The following are better preservatives: chloroform, toluene, ether and thymol.

(5) The best preservatives are salicylic acid, strychnine sulfate, sodium arsenite and probably sandalwood oil.

SEATTLE, WASE.

[From the Chemical Section of the Iowa Agricultural Experiment Station.]

\title{
THE EFFECT OF ACIDS AND ALRALIES UPON THE CATALASE OF TAKA-DIASTASE.
}

BY RAY E. NEIDIG.

Received December 22, 1913.

It is well known that extracts from plant and animal tissues contain catalase, an enzyme capable of decomposing hydrogen peroxide. The effect of acids and bases toward this enzyme has been the subject of numerous investigations. In this field, Jacobson ${ }^{1}$ was the first to note that acid caused a retarding effect upon solutions of catalase obtained from blood. Later, Senter ${ }^{2}$ made a more exhaustive study of blood catalase, in which he determined the effect of three acids, namely, hydrochloric, nitric and acetic, and also of sodium hydroxide upon this enzyme. From this investigation he concluded that the time of contact of the acid with the catalase solution had very little influence upon the reaction, that there was no decomposition of the enzyme during its contact with acid, and, lastly, that neutralization of the acid restored the original activity of the cátalase. Sodium hydroxide, he found, exercised an inhibiting effect similar to that of acids.

As these first two investigators dealt wholly with catalase from blood, Euler $^{3}$ undertook an investigation to determine whether or not catalase from different sources was identical. His work included catalase from one of the higher fungi, viz., Boletus scaber. He found an important difference between the action of acids on the inhibition of the catalase obtained from Boletus scaber and that obtained from blood (Senter), ${ }^{4}$ tobacco extract (Loew), ${ }^{5}$ and yeast (Issaew). ${ }^{6}$. He also observed that freshly precipitated magnesium hydroxide greatly increased the catalytic power of the enzyme. In this observation he corroborated the work of Loew, ${ }^{4}$ who noted the same increase in enzymic activity when magnesium hydroxide was added to extracts of tobacco.

\footnotetext{
${ }^{1} Z$. physiol. Chem., 16, 340.

${ }^{2}$ Ibid., 44, 257 (1903).

${ }^{3}$ Hofm. Beitr., 7, 1 (1905).

1 Loc. cit.

' U. S. Dept. of Agr., Report No. 68.

${ }^{6} Z$. physiol. Chem., 42, 102 (I904).
} 
In view of the results obtained by Euler, the writer thought it of interest to study the effect of a number of the more important acids and alkalies upon catalase obtained from lower fungi. It has been shown by Bach and Chodat that cultures of Sterigmatocystis nigra contain catalase. Pringsheim ${ }^{2}$ demonstrated catalase in the pressed juice of fifteen out of seventeen molds studied by him. Dox ${ }^{3}$ has shown that catalase was present in the filtrate of thirty-four out of thirty-five molds which he examined, and Loew ${ }^{4}$ observed that the filtrate from Pencillium glaucum showed the power of decomposing hydrogen peroxide, and also that the commercial taka-diastase, an enzyme preparation of high purity made from the fungus Aspergillus oryzae, is also rich in catalase. It occurred to the writer that, owing to the high degree of purity of the commercial taka-diastase, this enzyme preparation would be an excellent source of material to use in a study on the catalase of lower fungi.

\section{Experimental.}

'The methods and apparatus used in determining the strength of catalase are numerous and will not be reviewed in detail here. 'The principle on which the methods are based is the determination of the amount of free oxygen liberated when a solution containing catalase is brought in contact with hydrogen peroxide. This is commonly performed by collecting over water the oxygen given off and reducing the results to $0^{\circ}$ and $760 \mathrm{~mm}$. pressure, or by measuring the amount of pressure developed by means of a manometer, and lastly, by titrating the undecomposed hydrogen peroxide with a standard solution of potassium permanganate.

Van Slyke's amino nitrogen apparatus was used in the determinations, and found to be very satisfactory where the enzyme solution is comparatively pure. The advantages of this apparatus over many others are: (I) its ease of manipulation, and (2) the uniformity of results obtained by maintaining a constant rate of agitation during the reaction between enzyme and peroxide. That the speed of shaking has a direct influence upon the amount of oxygen liberated during a given time was previously shown by Loew and confirmed by the writer in the following experiment:

To each of two equal portions of enzyme solution in the apparatus were added three cubic centimeters of hydrogen peroxide. The total oxygen given off was noted at the end of ten minutes:
(I) with shaking..
$33.6 \mathrm{cc}$. oxygen
(2) withont shaking.
$23.5 \mathrm{cc}$. oxygen

While shaking the enzyme solution the apparatus averaged I40 oscillations per minute. The same speed was maintained as nearly as possible throughout the following determinations:

\footnotetext{
1 Ber., 36, 1756 (1893).

"Z. physiol. Chem., 62, 386 (1909).

"This Journal, 32 (Oct., Igro).

${ }^{4}$ Loc. cit.
} 
The enzyme solution used in this work was prepared by dissolving 0.625 gram of the commercial taka-diastase in Ioo cc. of water. After filtering, ten cc. of this solution were diluted so that, when the required amount of acid was added, the total volume of the solution was ${ }_{5} \mathrm{cc}$. Throughout the work, unless otherwise stated, the time of contact of an acid with the enzyme solution was fifteen minutes and at the end of that time the solution was diluted with distilled water to $22 \mathrm{cc}$. and then brought in contact with 3 cc. of hydrogen peroxide. The volume of oxygen developed was read at the end of each minute for a period of ten minutes. As a control experiment the same procedure was followed except that the acid was omitted. A similar experiment was made to ascertain the effect of neutralization of the acid on the enzyme activity after fifteen minutes' contact with the enzyme solution. This was accomplished by adding to the acid solution the exact required amount of standard sodium hydroxide, then making the volume up to $22 \mathrm{cc}$. and mixing the solution with $3 \mathrm{cc}$. hydrogen peroxide, noting the volume of oxygen developed each minute for a period of ten minutes. The tables follow:

TABLE I. ${ }^{1}$

Effect of I5-Minute Contact with o. $\mathrm{N} \mathrm{H}_{2} \mathrm{SO}_{4}$.

\begin{tabular}{|c|c|c|c|c|c|c|c|}
\hline \multirow{2}{*}{$\begin{array}{c}\text { Time after } \\
\text { adding } \\
\mathrm{HaO}_{2} \\
\text { (Minutes.) }\end{array}$} & \multicolumn{7}{|c|}{$0.1 \mathrm{~N} \mathrm{H}_{2} \mathrm{SO}_{4}$ added. } \\
\hline & $0 \mathrm{cc}$ & $0.2 \mathrm{cc}$ & $0.4 \mathrm{cc}$ & $\begin{aligned} & 0.8 \mathrm{cc} \\
& \mathrm{en} \text { deve }\end{aligned}$ & $\begin{array}{l}\text { d. } \\
\text { d. }\end{array}$ & $1.4 \mathrm{cc}$. & $1.6 \mathrm{cc}$ \\
\hline I....... & 9.2 & 8.6 & 8.0 & 4.6 & $I \cdot 3$ & trace & 0.0 \\
\hline $2 \ldots \ldots$ & I5.9 & 15.4 & 14.0 & 9.7 & 3.2 & 0.3 & 0.0 \\
\hline $3 \ldots \ldots$ & 20.7 & 19.6 & 18.3 & $\mathrm{I} 3.8$ & $4 \cdot 4$ & 0.4 & 0.0 \\
\hline $4 \ldots \ldots$ & $24 \cdot 5$ & 22.9 & $2 I \cdot 3$ & I6.6 & $5 \cdot I$ & 0.5 & 0.0 \\
\hline $5 \ldots \ldots \ldots$ & 26.6 & 25.0 & $23 \cdot 4$ & 18.6 & 5.6 & 0.6 & 0.0 \\
\hline $6 \ldots \ldots$ & 28.1 & 26.9 & 25.0 & 19.9 & 5.9 & 0.7 & 0.0 \\
\hline $7 \ldots \ldots$ & 29.4 & 28.4 & 26.4 & 20.8 & 6.3 & 0.8 & 0.0 \\
\hline $8 \ldots \ldots$ & 30.3 & 29.5 & 27.4 & $21 \cdot 5$ & 6.6 & 0.8 & 0.0 \\
\hline $9 \ldots \ldots$ & $3 \mathrm{I} \cdot \mathrm{I}$ & 30.5 & 28.3 & 22.0 & 6.6 & 0.8 & 0.0 \\
\hline 10....... & $3 \mathrm{I} .8$ & 31.2 & 28.9 & 22.4 & 6.7 & 0.8 & 0.0 \\
\hline
\end{tabular}

Effect of Neutralization of $\mathrm{H}_{2} \mathrm{SO}_{4}$ after ${ }_{15}$-Minute Contact.

$\begin{array}{rrrrrrrr}1 \ldots \ldots & 9.2 & 9.2 & 7.9 & 5.3 & 1.4 & \text { trace } & 0.0 \\ 2 \ldots \ldots & 15.9 & 15.9 & 14.6 & 11.2 & 3.8 & 0.5 & 0.0 \\ 3 \ldots \ldots & 20.7 & 20.9 & 19.2 & 15.0 & 5.5 & 1.0 & 0.0 \\ 4 \ldots \ldots & 24.5 & 23.7 & 22.4 & 17.4 & 6.5 & 1.2 & 0.0 \\ 5 \ldots \ldots & 26.6 & 25.7 & 24.6 & 18.8 & 7.8 & 1.5 & 0.0 \\ 6 \ldots \ldots & 28.1 & 27.7 & 26.4 & 20.1 & 8.3 & 1.8 & 0.0 \\ 7 \ldots \ldots & 29.4 & 28.9 & 27.7 & 21.1 & 8.8 & 2.0 & 0.0 \\ 8 \ldots \ldots & 30.3 & 30.0 & 28.7 & 22.0 & 9.1 & 2.2 & 0.0 \\ 9 \ldots \ldots & 31.1 & 30.8 & 29.7 & 22.6 & 9.3 & 2.4 & 0.0 \\ 10 \ldots \ldots & 31.8 & 31.5 & 30.5 & 23.2 & 9.5 & 2.5 & 0.0\end{array}$

${ }^{1}$ All the volumes given in this paper are reduced to $0^{\circ}$ and $760 \mathrm{~mm}$. pressure. volume.

${ }^{2}$ The hydrogen peroxide used contained $3.8 \mathrm{I} \%$ oxygen by weight or $12.54 \%$ by 
The results are illustrated graphically by the following curves: Fig. I illustrates the rate of liberation of oxygen during the first ten minutes, after 15 -minutes contact with hydrogen peroxide and for the various de-
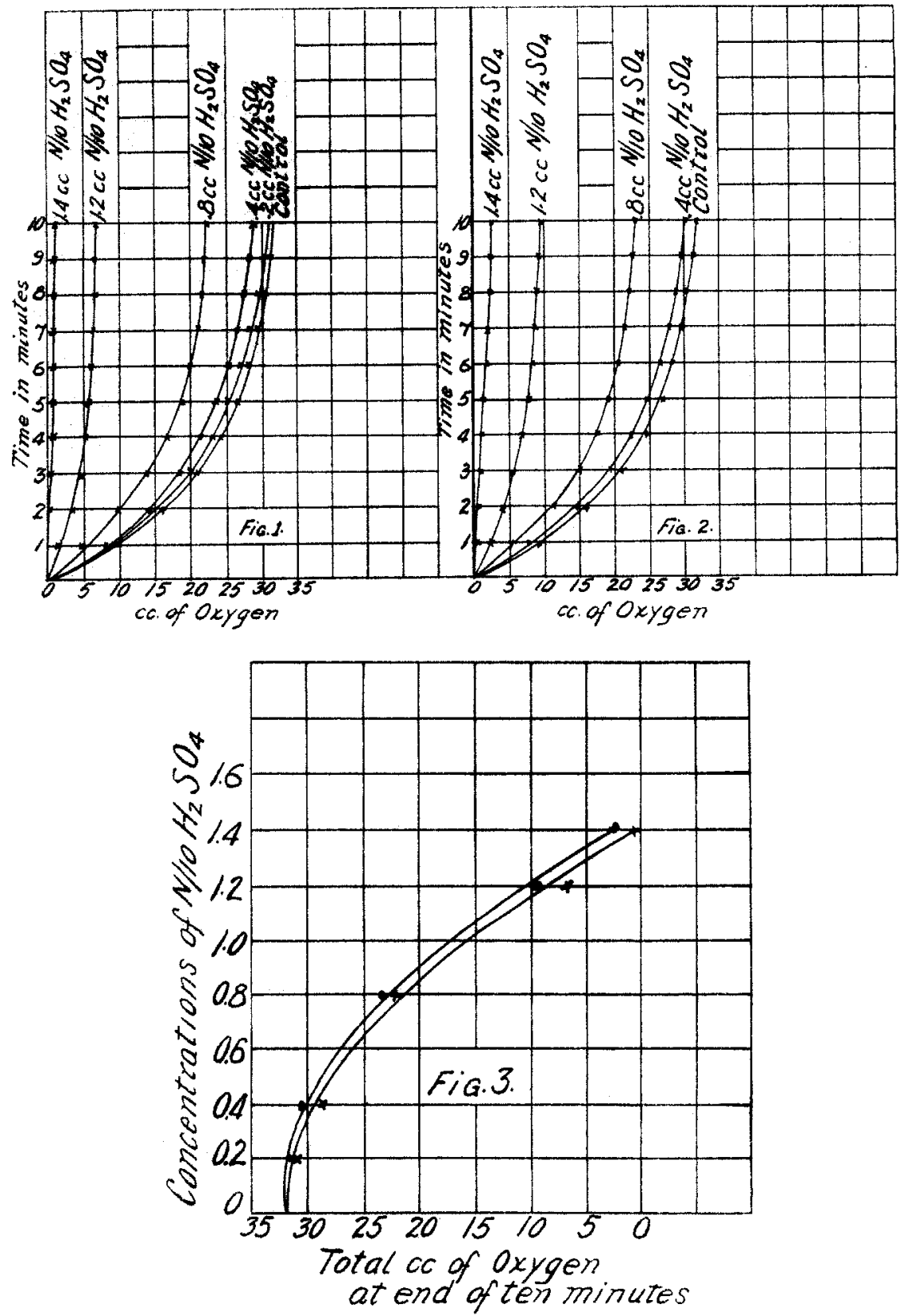
TABLE II.

Effect of 15-Minute Contact with o.I $N \mathrm{HCl}$.

\begin{tabular}{|c|c|c|c|c|c|c|c|}
\hline \multirow{2}{*}{$\begin{array}{l}\text { Time after } \\
\text { adding } \\
\mathrm{H}_{2} \mathrm{O}_{2} \text {. } \\
\text { (Minutes.) }\end{array}$} & \multicolumn{7}{|c|}{$0.1 \mathrm{~N} \mathrm{HCl}$ added. } \\
\hline & $0 \mathrm{cc}$ & $0.2 \mathrm{cc}$ & \multicolumn{3}{|c|}{$\begin{array}{l}0.4 \mathrm{cc} \text {. } \\
\text { Oxygen developed. }\end{array}$} & $1.4 \mathrm{cc}$. & $1.6 \mathrm{cc}$. \\
\hline $\mathbf{I} \ldots \ldots \ldots$ & 8.8 & 7.8 & 7.6 & 2.5 & 0.9 & trace & 0.0 \\
\hline $2 \ldots \ldots$ & I5. 9 & 14.9 & I5 .8 & $7 \cdot 2$ & $2 \cdot 5$ & trace & 0.0 \\
\hline $3 \ldots \ldots \ldots$ & 21.0 & 20.0 & 20.7 & 10.8 & 3.6 & trace & 0,0 \\
\hline $4 \ldots \ldots$ & 25.0 & 23.6 & $24 \cdot I$ & 13.9 & $4 \cdot 7$ & 0.3 & 0.0 \\
\hline $5 \ldots \ldots \ldots$ & $27 \cdot 4$ & 26.1 & 26.3 & I6. I & $5 \cdot 5$ & 0.4 & 0.0 \\
\hline $6 \ldots \ldots$ & $29 \cdot 3$ & 28.0 & 28.1 & I $7 \cdot 7$ & 6.3 & 0.4 & 0.0 \\
\hline $7 \ldots \ldots$ & 30.8 & $29 \cdot 3$ & 29.4 & I9. I & 6.9 & 0.5 & 0.0 \\
\hline $8 \ldots \ldots \ldots$ & $3 \mathrm{I} .8$ & 30.3 & 30.6 & 20.2 & $7 \cdot 4$ & 0.6 & 0.0 \\
\hline $9 \ldots \ldots$ & 32.6 & 31.2 & $3 \pi \cdot 5$ & $2 \mathrm{I} \cdot \mathrm{I}$ & $7 \cdot 9$ & 0.7 & 0.0 \\
\hline Io....... & $33 \cdot 2$ & 31.8 & 32.0 & 21.8 & 8.3 & 0.8 & 0.0 \\
\hline \multicolumn{8}{|c|}{ Effect of Neutralization of $\mathrm{HCl}$ after 15-Minute Contact. } \\
\hline I $\ldots \ldots$ & 8.8 & 8.0 & 7.6 & 3.6 & $2 . I$ & 0.0 & 0.0 \\
\hline $2 \ldots \ldots$ & I5.9 & 15.2 & 14.9 & $9 \cdot I$ & 4.8 & trace & 0.0 \\
\hline $3 \ldots \ldots$ & 21.0 & 20.3 & 20.3 & $13 \cdot 7$ & $7 \cdot 3$ & trace & 0.0 \\
\hline $4 \ldots \ldots \ldots$ & 25.0 & 23.6 & 23.9 & 17.2 & $9 \cdot 5$ & 0.4 & 0.0 \\
\hline $5 \ldots \ldots$ & $27 \cdot 4$ & 26.1 & 26.5 & 20.0 & I I. 6 & 0.5 & 0.0 \\
\hline $6 \ldots \ldots$ & $29 \cdot 3$ & 28.0 & 28.1 & 21.8 & $13 \cdot 2$ & 0.7 & 0.0 \\
\hline $7 \ldots \ldots$ & 30.8 & $29 \cdot 3$ & 29.5 & 23.6 & I 4.4 & 0.9 & 0.0 \\
\hline $8 \ldots \ldots$ & 31.8 & 30.3 & 30.6 & 24.9 & $15 \cdot 5$ & 1.0 & 0.0 \\
\hline $9 \ldots \ldots$ & 32.6 & $3 \mathbf{I} .1$ & $3 I .5$ & 25.9 & 16.4 & 1.0 & 0.0 \\
\hline 10....... & 33.2 & 31.8 & 32.2 & 26.8 & 17.0 & I. I & 0.0 \\
\hline
\end{tabular}

TABLE III.

Effect of 15-Minute Contact with o.1 $N$ Oxalic Acid.

\begin{tabular}{|c|c|c|c|c|c|c|c|}
\hline \multirow{2}{*}{$\begin{array}{l}\text { Time after } \\
\text { adding } \\
\mathrm{H}_{2} \mathrm{O}_{2 .} \\
\text { (Minutes.) }\end{array}$} & \multicolumn{7}{|c|}{$0.1 \mathrm{~N}$ oxalic acid added. } \\
\hline & $0 \mathrm{cc}$ & $0.4 \mathrm{cc}$ & $0.8 \mathrm{cc}$ & $\begin{array}{l}1.6 \mathrm{cc} . \\
\text { en devel }\end{array}$ & $2 \mathrm{cc}$ & $2.4 \mathrm{cc}$ & $2.6 \mathrm{cc}$. \\
\hline $\mathbf{1} \ldots \ldots \ldots$ & 8.9 & 8.2 & 6.5 & $3 \cdot 4$ & 0.7 & trace & 0.0 \\
\hline $2 \ldots \ldots \ldots$ & I5.9 & 15.8 & I2. 6 & 7.0 & I. 8 & 0.2 & 0.0 \\
\hline $3 \ldots \ldots \ldots$ & 20.8 & 21.3 & 17.0 & 9.6 & 2.8 & 0.4 & 0.0 \\
\hline $4 \ldots \ldots \ldots$ & $24 . I$ & 24.1 & 19.7 & I I .5 & 3.8 & 0.5 & trace \\
\hline $5 \ldots \ldots \ldots$ & 26.0 & 26.0 & $21 \cdot 5$ & 12.9 & $4 \cdot 7$ & 0.7 & trace \\
\hline $6 \ldots \ldots \ldots$ & $27 \cdot 3$ & $27 \cdot 7$ & 22.8 & 14.0 & $5 \cdot 5$ & 0.9 & trace \\
\hline $7 \ldots \ldots \ldots$ & 28.5 & 29.0 & 23.8 & $14 \cdot 9$ & 6.2 & 1.0 & trace \\
\hline $8 \ldots \ldots \ldots \ldots$ & 29.5 & 30.0 & 24.6 & $15 \cdot 5$ & 6.7 & I . I & trace \\
\hline $9 \ldots \ldots \ldots$ & 30.4 & 31.0 & $25 \cdot 3$ & 16.0 & $7 \cdot 3$ & I. 2 & 0.4 \\
\hline $10 \ldots . . .$. & $3 I . I$ & $31 \cdot 7$ & 25.8 & 16.4 & 7.6 & $1 \cdot 3$ & 0.4 \\
\hline \multicolumn{8}{|c|}{ Effect of Neutralization of Oxalic Acid after I5-Minute Contact. } \\
\hline $\mathbf{I} \ldots \ldots \ldots$ & 8.9 & 8.4 & $7 \cdot 3$ & $5 \cdot 3$ & $2, I$ & 0.7 & 0.0 \\
\hline $2 \ldots \ldots \ldots \ldots$ & I5.9 & 15.8 & I4. I & 10.3 & 3.9 & 1.7 & 0.0 \\
\hline $3 \ldots \ldots \ldots$ & 20.8 & 20.1 & 18.5 & 13.9 & $5 \cdot 7$ & 2.6 & 0.0 \\
\hline $4 \ldots \ldots \ldots$ & 24.1 & 23.1 & 21.4 & 16.1 & $7 \cdot 2$ & $3 \cdot 4$ & trace \\
\hline $5 \ldots \ldots \ldots \ldots$ & 26.0 & 25.0 & $23 \cdot 4$ & $17 \cdot 5$ & 8.7 & $4 \cdot 2$ & trace \\
\hline $6 \ldots \ldots \ldots$ & $27 \cdot 3$ & 26.4 & 24.8 & 18.5 & 9.9 & 4.8 & trace \\
\hline $7 \ldots \ldots \ldots$ & 28.5 & $27 \cdot 4$ & $25 \cdot 9$ & 19.4 & 10.9 & $5 \cdot 3$ & trace \\
\hline $8 \ldots \ldots \ldots \ldots$ & 29.5 & 28.3 & 26.9 & 20.1 & I I. 7 & $5 \cdot 7$ & trace \\
\hline $9 \ldots \ldots \ldots$ & 30.4 & 29.0 & $27 \cdot 4$ & 20.6 & 12.4 & 6.0 & trace \\
\hline Iо. . . . . . . & $3 I . I$ & 29.5 & 28.0 & 2 I. I & 13.0 & 6.3 & trace \\
\hline
\end{tabular}


TABLE IV.

Effect of 15 -Minute Contact with Normal Tartaric Acid.

Time after adding $\mathrm{H}_{2} \mathrm{O}_{2}$.

(Minutes)
Nornal tartaric acid added.

\begin{tabular}{rrrrrr}
$10.1 \mathrm{cc}$ & \multicolumn{2}{c}{$0.2 \mathrm{cc}$} & $0.4 \mathrm{cc}$ & $0.6 \mathrm{cc}$ & $0.8 \mathrm{cc}$. \\
9.1 & 7.8 & 4.4 & 2.7 & 0.8 & trace \\
15.7 & 14.7 & 9.3 & 6.1 & 1.7 & 0.2 \\
20.2 & 18.7 & 13.0 & 8.4 & 2.8 & 0.5 \\
23.4 & 21.0 & 15.5 & 9.8 & 3.6 & 0.7 \\
25.6 & 22.8 & 16.9 & 10.8 & 4.3 & 0.8 \\
27.2 & 24.0 & 18.0 & 11.4 & 4.8 & 0.8 \\
28.5 & 24.8 & 18.8 & 11.9 & 5.2 & 0.9 \\
29.5 & 25.7 & 19.6 & 12.2 & 5.6 & 0.9 \\
30.2 & 26.5 & 19.9 & 12.5 & 5.8 & 0.9 \\
31.0 & 26.8 & 20.3 & 12.7 & 6.1 & 0.9
\end{tabular}

Effect of Neutralization of Tartaric Acid after 15 -Minute Contact.

\begin{tabular}{|c|c|c|c|c|c|c|}
\hline $1 \ldots \ldots \ldots$ & $9 \cdot 1$ & 7.8 & $5 \cdot 3$ & $5 \cdot 4$ & I. 3 & 0.8 \\
\hline $2 \ldots \ldots \ldots$ & $15 \cdot 7$ & 15.2 & 10.6 & 8.8 & 2.7 & 1.0 \\
\hline $3 \ldots \ldots \ldots$ & 20.2 & 20.2 & I $4 \cdot 4$ & II, I & 3.9 & I. 3 \\
\hline $4 \ldots \ldots \ldots$ & $23 \cdot 4$ & $21 \cdot 7$ & $17 \cdot 2$ & 12.8 & $4 \cdot 9$ & 1.7 \\
\hline $5 \ldots \ldots \ldots$ & $25 \cdot 6$ & $23 \cdot 3$ & 19.1 & 14.0 & $5 \cdot 7$ & I. 9 \\
\hline $6, \ldots \ldots \ldots$ & $27 \cdot 2$ & 24.6 & 20.6 & 14.8 & 6.4 & I. 9 \\
\hline $7 \ldots \ldots \ldots$ & 28.5 & $25 \cdot 4$ & 21.8 & I $5 \cdot 5$ & $7 \cdot 1$ & $2 . I$ \\
\hline $8 \ldots \ldots \ldots$ & $29 \cdot 5$ & 26.2 & 22.7 & I6, I & 7.6 & 2.2 \\
\hline $9 \ldots \ldots \ldots$ & 30.2 & 26.9 & $23 \cdot 5$ & 16.5 & $8 . \mathrm{I}$ & $2 \cdot 3$ \\
\hline $10 \ldots . . .$. & 31.0 & $27 \cdot 4$ & $24, I$ & 16.9 & 8.6 & 2.3 \\
\hline
\end{tabular}

TABLE $V$.

Effect of 15-Minute Contact with Normal Citric Acid.

\begin{tabular}{|c|c|c|c|c|c|c|c|}
\hline \multirow{2}{*}{$\begin{array}{l}\text { Time after } \\
\text { adding } \\
\mathrm{H}_{2} \mathrm{O}_{2} \\
\text { (Minutes.) }\end{array}$} & \multicolumn{7}{|c|}{ Normal citric acid added. } \\
\hline & $0 \mathrm{cc}$ & $0.2 \mathrm{cc}$ & $0.4 \mathrm{cc}$ & $\begin{array}{l}0.8 \mathrm{cc} \text {. } \\
\text { en devel }\end{array}$ & $\begin{array}{l}1.2 \mathrm{cc} \\
\text { ed. }\end{array}$ & $1.6 \mathrm{cc}$ & $1.8 \mathrm{cc}$ \\
\hline $1, \ldots \ldots \ldots$ & 10.3 & 6.6 & 5.0 & 2.9 & 1.0 & trace & 0.0 \\
\hline $2 \ldots \ldots \ldots \ldots$ & 16.6 & 13.2 & 9.9 & 6.0 & 2.0 & 0.3 & 0.0 \\
\hline $3 \ldots \ldots \ldots$ & 20.7 & $17 \cdot 7$ & I $3 \cdot 5$ & $7 \cdot 4$ & 3.1 & 0.5 & 0.0 \\
\hline $4 \ldots \ldots \ldots$. & 23.8 & 20.1 & I5.7 & 8.3 & 4.1 & 0.8 & 0.0 \\
\hline $5 \ldots \ldots \ldots$ & $25 \cdot 7$ & 21.8 & 17.2 & 8.9 & $4 \cdot 9$ & 0.9 & 0.0 \\
\hline $6 \ldots \ldots \ldots$ & $27 \cdot 3$ & 23.0 & I 8.3 & $9 \cdot 3$ & $5 \cdot 5$ & I.O & 0.0 \\
\hline $7 \ldots \ldots \ldots$ & 28.4 & $23 \cdot 9$ & 19.0 & 9.6 & $6 . \mathrm{I}$ & 1.0 & trace \\
\hline $8 \ldots \ldots \ldots \ldots$ & 29.2 & $24 \cdot 7$ & 19.6 & 9.8 & 6.7 & I. I & trace \\
\hline $9 \ldots \ldots \ldots$ & 30.0 & 25.2 & 20.1 & $9 \cdot 9$ & 7.0 & I. 2 & trace \\
\hline 10.......... & 30.7 & $25 \cdot 7$ & 20.4 & Io. I & $7 \cdot 3$ & I.3 & trace \\
\hline \multicolumn{8}{|c|}{ Effect of Neutralization of Citric Acid after 15-Minute Contact. } \\
\hline $1 \ldots \ldots \ldots \ldots$ & 10.3 & 8.4 & 6.5 & $4 \cdot 2$ & I. I & trace & trace \\
\hline $2 \ldots \ldots \ldots$ & 16.6 & 14.6 & 11.8 & $7 \cdot 9$ & 2.7 & 0.3 & trace \\
\hline $3 \ldots \ldots \ldots$ & 20.7 & 18.5 & I5.I & 10.2 & 3.8 & 0.5 & 0.2 \\
\hline $4 \ldots \ldots \ldots$ & 23.8 & 20.9 & $17 \cdot 4$ & II. 7 & 4.8 & 0.6 & 0.3 \\
\hline $5 \ldots \ldots \ldots$ & $25 \cdot 7$ & $22 \cdot 4$ & 18.9 & 12.7 & 5.8 & 0.8 & 0.3 \\
\hline $6 \ldots \ldots \ldots$ & $27 \cdot 3$ & 23.0 & 20.0 & $\mathrm{r} 3 \cdot 4$ & 6.7 & 1.0 & 0.4 \\
\hline $7 \ldots \ldots \ldots$ & 28.4 & 24.6 & 20.8 & 13.9 & $7 \cdot 3$ & 1.0 & 0.7 \\
\hline $8 \ldots \ldots \ldots$ & 29.2 & $25 \cdot 2$ & 21.4 & $I 4 \cdot 3$ & 7.8 & $I, I$ & 0.5 \\
\hline $9 \ldots \ldots \ldots$ & 30.0 & 25.8 & 21.9 & 14.6 & 8.1 & 1.2 & 0.5 \\
\hline $10 \ldots \ldots \ldots$ & 30.7 & 26.4 & $22 \cdot 3$ & I5.0 & 8.4 & I. 3 & 0.6 \\
\hline
\end{tabular}


TABLE VI.

Effect of 15-Minute Contact with Normal Acetic Acid.

\begin{tabular}{|c|c|c|c|c|c|c|c|}
\hline \multirow{2}{*}{$\begin{array}{l}\text { Time after } \\
\text { adding } \\
\mathrm{H}_{2} \mathrm{O}_{2} \\
\text { (Minutes.) }\end{array}$} & \multicolumn{7}{|c|}{ Normal acetic acid added. } \\
\hline & $0 \mathrm{cc}$. & $0.5 \mathrm{cc}$. & $1.5 \mathrm{cc}$. & $\begin{array}{l}4.5 \mathrm{cc} \\
\text { in dev }\end{array}$ & $\begin{array}{l}6 \mathrm{cc} . \\
\text { d. }\end{array}$ & $8 \mathrm{cc}$ & $10 \mathrm{cc}$. \\
\hline $1 \ldots \ldots \ldots \ldots$ & 9.0 & 4.2 & 2.4 & 0.6 & 0.3 & trace & 0.0 \\
\hline $2 \ldots \ldots \ldots$ & 16.2 & 9.6 & 6.4 & 2.2 & r.o & 0.3 & 0.0 \\
\hline $3 \ldots \ldots \ldots$ & $2 \mathrm{I} .7$ & 14.0 & 9.3 & 3.6 & I. 2 & 0.4 & 0.0 \\
\hline $4 \ldots \ldots \ldots$ & 25.1 & 17.3 & I2. I & $4 \cdot 7$ & I. 5 & 0.7 & trace \\
\hline $5 \ldots \ldots \ldots$ & 27.8 & 20.0 & \pm 3.8 & $4 \cdot 5$ & $\mathrm{x} .8$ & 0.9 & trace \\
\hline $6 \ldots \ldots \ldots$ & 29.7 & 22.0 & I5.9 & 6.3 & 2.2 & I.O & 0.2 \\
\hline $7 \ldots \ldots \ldots$ & $3 \mathrm{r} \cdot \mathrm{I}$ & 23.4 & 17.2 & 7.0 & 2.5 & I. I & 0.3 \\
\hline $8 \ldots \ldots \ldots$ & 32.1 & 24.6 & 18.4 & $7 \cdot 7$ & 2.9 & $\mathrm{I} .2$ & 0.4 \\
\hline $9 \ldots \ldots \ldots$ & 32.9 & 25.6 & r9.4 & 8.4 & 3.1 & I. 3 & 0.4 \\
\hline ro.......... & $33 \cdot 5$ & 26.4 & 20.3 & 8.9 & 3.4 & I. 4 & 0.4 \\
\hline
\end{tabular}

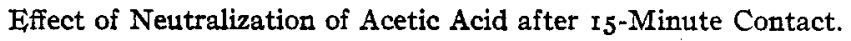

$\begin{array}{rrrrrrrr}1 \ldots \ldots \ldots \ldots & 9.0 & 6.9 & 6.9 & 4.9 & 2.0 & 1.4 & 1.2 \\ 2 \ldots \ldots \ldots & 16.2 & 13.3 & 12.4 & 8.4 & 5.1 & 3.2 & 2.3 \\ 3 \ldots \ldots \ldots \ldots & 21.7 & 19.8 & 16.7 & 11.7 & 7.6 & 4.7 & 3.3 \\ 4 \ldots \ldots \ldots & 25.1 & 23.3 & 20.0 & 13.8 & 9.3 & 5.8 & 4.0 \\ 5 \ldots \ldots \ldots \ldots & 27.8 & 25.5 & 21.9 & 15.5 & 10.5 & 6.6 & 4.7 \\ 6 \ldots \ldots \ldots & 29.7 & 26.9 & 23.1 & 16.6 & 11.5 & 7.2 & 5.2 \\ 7 \ldots \ldots \ldots \ldots & 31.1 & 27.5 & 24.2 & 17.3 & 12.2 & 7.7 & 5.7 \\ 8 \ldots \ldots \ldots \ldots & 32.1 & 29.0 & 25.1 & 18.0 & 12.7 & 8.0 & 6.1 \\ 9 \ldots \ldots \ldots \ldots & 32.9 & 29.9 & 25.9 & 18.5 & 13.1 & 8.3 & 6.3 \\ 10 \ldots \ldots \ldots & 33.5 & 30.9 & 26.5 & 19.0 & 13.5 & 8.4 & 6.4\end{array}$

grees of acid concentrations. The curves shown in Fig. 2 are analogous to those in Fig. I except that the determination was made after exactly neutralizing with sodium hydroxide.

Curve $a$, Fig. 3 shows the total volume of oxygen developed during the first ten minutes after 15 -minute contact with different acid concentrations. Curve $b$ in the same figure represents similar determinations after neutralizing the acid with sodium hydroxide. A comparison of these two curves shows that the effect of neutralization is very slight. The curves for the remaining acids show the same general characteristics and will not be included here.

The foregoing experiments show the relative inhibiting effect of acids toward catalase, when in contact with the enzyme solution for fifteen minutes. In order to ascertain the inhibiting effect of acids, when brought in contact with the enzyme solution for different periods of time, the following experiments were performed:

Different strengths of two of the inorganic acids were allowed to remain in contact with the taka-diastase solution for $0,15,30$, and 60 minutes. The acids used were decinormal sulfuric $(0.8 \mathrm{cc}$.) and hydrochloric ( 0.4 cc.), respectively. The activities of the catalase solutions were then determined in the usual manner. 
TABLE VII.

Effect of 0.4 ce. $0.1 \mathrm{~N} \mathrm{HCl}$ for Different Periods of Contact.

\begin{tabular}{|c|c|c|c|c|c|}
\hline \multirow{2}{*}{$\begin{array}{l}\text { Time } \\
\text { after } \\
\text { adding } \\
\text { HiO.. } \\
\text { (Minutes.) }\end{array}$} & \multirow[b]{2}{*}{ Control. } & \multicolumn{4}{|c|}{$\begin{array}{l}0.4 \mathrm{cc}, 0.1 \mathrm{~N} \mathrm{FCl} \text { added. } \\
\text { Time of contact in minutes. }\end{array}$} \\
\hline & & \multicolumn{3}{|c|}{$\begin{array}{l}15 . \\
\text { Oxygen developed. }\end{array}$} & 60. \\
\hline I. $\ldots \ldots \ldots \ldots \ldots \ldots$ & $7 \cdot 9$ & $5 . I$ & 5.2 & 4.9 & $5 \cdot I$ \\
\hline $2 \ldots \ldots \ldots \ldots \ldots \ldots$ & 14.2 & 10.9 & 11.0 & 10.8 & 10.3 \\
\hline $3 \ldots \ldots \ldots \ldots \ldots \ldots$ & 18.5 & 15.2 & 15.6 & $15 \cdot 5$ & I5. I \\
\hline $4 \ldots \ldots \ldots \ldots \ldots \ldots$ & 21.8 & $\mathrm{r} 8.6$ & 19.2 & 18.7 & 18.7 \\
\hline $5 \ldots \ldots \ldots \ldots \ldots \ldots$ & $24 \cdot 5$ & 21.8 & 21.8 & $22 . x$ & 21.2 \\
\hline $6 \ldots \ldots \ldots \ldots \ldots \ldots$ & 26.7 & 24.1 & 24.0 & 23.9 & 23.6 \\
\hline $\begin{array}{l}7 \ldots \ldots \ldots \ldots \ldots \ldots \ldots \ldots \ldots \ldots \\
8 \ldots \ldots \ldots \ldots \ldots \ldots \ldots\end{array}$ & $\begin{array}{l}29.3 \\
29.8\end{array}$ & $\begin{array}{l}25.6 \\
26.8\end{array}$ & $\begin{array}{l}25 \cdot 7 \\
27.0\end{array}$ & $\begin{array}{l}25.6 \\
26.7\end{array}$ & $\begin{array}{l}25.6 \\
26.9\end{array}$ \\
\hline $9 \ldots \ldots \ldots \ldots \ldots \ldots$ & 30.9 & 27.9 & 28.2 & 28.1 & 28.0 \\
\hline 10 $\ldots \ldots \ldots \ldots \ldots \ldots$ & 31,8 & 28.9 & 29.0 & 29.0 & 28.7 \\
\hline
\end{tabular}

TABLE VIII.

Effect of 0.8 cc. $0.1 \mathrm{~N} \mathrm{H}_{2} \mathrm{SO}_{4}$ for Different Periods of Contact.

\begin{tabular}{|c|c|c|c|c|c|}
\hline \multirow{2}{*}{$\begin{array}{l}\text { Time } \\
\text { after } \\
\text { adding } \\
\text { HaOng. } \\
\text { (Minutes.) }\end{array}$} & \multirow[b]{2}{*}{ Control. } & \multicolumn{4}{|c|}{ 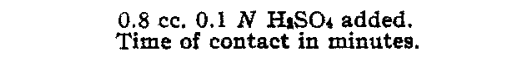 } \\
\hline & & 0. & $\begin{array}{l}15 . \\
0 \times y\end{array}$ & $\begin{array}{l}30 . \\
\text { veloped. }\end{array}$ & 60. \\
\hline $1, \ldots \ldots \ldots \ldots \ldots$ & $7 \cdot 4$ & 2.8 & $2 . I$ & 2.1 & 2.0 \\
\hline $2 \ldots \ldots \ldots \ldots \ldots, \ldots$ & 13.7 & 6.9 & $5 \cdot 5$ & $5 \cdot 7$ & 4.8 \\
\hline $3 \ldots \ldots \ldots \ldots \ldots \ldots$ & 18.3 & 10.6 & 8.8 & 8.8 & $7 \cdot 5$ \\
\hline $4 \ldots \ldots \ldots \ldots \ldots \ldots \ldots$ & 22.1 & 13.1 & I I . 4 & II. 5 & IO. I \\
\hline $5 \ldots \ldots \ldots \ldots \ldots \ldots$ & $25 \cdot 5$ & $15 \cdot 4$ & $14 \cdot 3$ & 14.7 & II .8 \\
\hline $6, \ldots \ldots \ldots \ldots \ldots \ldots$ & 27.8 & 16.9 & 15.0 & 15.6 & $\mathrm{I} 4.0$ \\
\hline $7 \ldots \ldots \ldots \ldots \ldots \ldots$ & 29.6 & 18.4 & I 6.5 & 16.9 & 14.5 \\
\hline $8 \ldots \ldots \ldots \ldots \ldots \ldots$ & 30.9 & 19.3 & 17.4 & 18.1 & 15.8 \\
\hline $9 \ldots \ldots \ldots \ldots \ldots \ldots$ & 31.9 & 20.1 & 18.3 & 19.2 & I6. 5 \\
\hline $10 \ldots \ldots \ldots \ldots \ldots \ldots$ & 32.7 & 20.8 & 19.0 & 19.9 & 17.2 \\
\hline
\end{tabular}

The effect of acids toward the catalase of taka-diastase as shown by the preceding tables is very marked. In the series of acids studied none were found which did not show some inhibiting action. The inhibition, however, was not constant, equinormal solutions of different acids showing different power of inhibition toward the enzyme solution. The acids, arranged in order of the magnitude of their inhibiting effect, are hydrochloric, sulfuric, oxalic, tartaric, citric and acetic. It will be noted that this order corresponds to the order of their ionization constants.

Neutralization of the acid restored the enzyme activity only to a slight extent. In this respect catalase from taka-diastase differes from blood catalase. According to Senter, neutralization of the acid restores the original activity of the latter enzyme.

It has been shown above that the time, in which sulfuric and hydrochloric acids are in contact with the enzyme solution, has little or no effect upon the activity of catalase. The time of contact for the remaining 
TABLE IX.

Effect of I5-Minute Contact with Normal KOH.

Time after
adding
$\mathrm{H}_{2} \mathrm{O}_{2}$.
(Minutes.)

Normal $\mathrm{KOH}$ added.

\begin{tabular}{|c|c|c|c|c|c|c|}
\hline п. $\ldots \ldots \ldots \ldots \ldots$ & 8.2 & 6.4 & 4.I & I. 3 & 0.3 & 0.0 \\
\hline $2 \ldots \ldots \ldots \ldots \ldots \ldots$ & $\mathrm{I} 4 \cdot 4$ & 11.9 & 8.6 & 2.8 & 0.4 & 0.0 \\
\hline $3 \ldots \ldots \ldots \ldots \ldots$ & I8.9 & 16.3 & I $2 \cdot 5$ & 4.2 & 0.7 & trace \\
\hline $4 \ldots \ldots \ldots \ldots \ldots$ & $22 \cdot 3$ & 19.4 & I5. 4 & $5 \cdot 5$ & I. I & trace \\
\hline $5 \ldots \ldots \ldots \ldots \ldots \ldots$ & $24 \cdot 9$ & 22.0 & I $7 \cdot 7$ & 6.6 & I. 4 & 0.2 \\
\hline $6 \ldots \ldots \ldots \ldots \ldots \ldots$ & 27.1 & 23.8 & $19 \cdot 3$ & $7 \cdot 4$ & 1.6 & 0.3 \\
\hline $7 \ldots \ldots \ldots \ldots$ & 28.8 & 25.6 & 20.6 & 8. I & I. 7 & 0.3 \\
\hline $8 \ldots \ldots \ldots \ldots \ldots$ & 30.3 & 26.5 & $2 \mathrm{I} \cdot 7$ & 8.7 & I. 7 & 0.4 \\
\hline $9 \ldots \ldots \ldots \ldots \ldots$ & $3 I \cdot 3$ & $27 \cdot 5$ & $22 \cdot 5$ & 9.1 & I .8 & 0.4 \\
\hline $10 \ldots \ldots \ldots \ldots \ldots \ldots$ & $32 . I$ & 29.0 & $23 \cdot 3$ & $9 \cdot 5$ & I.9 & 0.4 \\
\hline
\end{tabular}

Effect of Neutralization of Normal KOH after I5-Minute Contact.

\begin{tabular}{|c|c|c|c|c|c|c|}
\hline 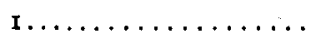 & 8.2 & 6.0 & $3 \cdot 9$ & 0.9 & trace & 0.0 \\
\hline $2 \ldots \ldots \ldots \ldots \ldots \ldots$ & I $4 \cdot 4$ & I I $\cdot 3$ & $7 \cdot 9$ & 2.5 & 0.2 & 0.0 \\
\hline $3 \ldots \ldots \ldots \ldots \ldots$ & 18.9 & 14.6 & 10.9 & $3 \cdot 5$ & 0.4 & trace \\
\hline $4 \ldots \ldots \ldots \ldots \ldots$ & $22 \cdot 3$ & 16.8 & 13.0 & $4 \cdot 3$ & 0.5 & trace \\
\hline $5 \ldots \ldots \ldots \ldots$ & $24 \cdot 9$ & I 8.8 & $14 \cdot 4$ & $4 \cdot 9$ & 0.6 & trace \\
\hline & 27.1 & 20.6 & I $5 \cdot 5$ & $5 \cdot 3$ & 0.7 & trace \\
\hline $7 \ldots \ldots \ldots \ldots \ldots$ & 28.8 & 22.0 & 16.4 & $5 \cdot 7$ & 0.8 & trace \\
\hline & 30.3 & $23 \cdot 2$ & $\mathrm{r} 7 \cdot \mathrm{I}$ & 6.0 & 0.9 & trace \\
\hline $9 \ldots \ldots \ldots \ldots \ldots$ & $3 I \cdot 3$ & 24.1 & I $7 \cdot 9$ & 6.2 & 0.9 & trace \\
\hline $10 \ldots \ldots \ldots \ldots \ldots$ & 32.1 & 24.9 & 18.5 & 6.4 & 0.9 & trace \\
\hline
\end{tabular}

TABLE X.

Effect of I5-Minute Contact with Normal NaOH.

\begin{tabular}{|c|c|c|c|c|c|c|}
\hline \multirow{2}{*}{$\begin{array}{l}\text { Time after } \\
\text { adding } \\
\mathrm{H}_{2} \mathrm{O}_{2 .} \\
\text { (Minutes.) }\end{array}$} & \multicolumn{6}{|c|}{ Normal NaOF added. } \\
\hline & $0 \mathrm{cc}$ & $0.1 \mathrm{cc}$ & $\begin{array}{c}0.2 \text { cc. } \\
\text { Oxygen }\end{array}$ & $\begin{array}{l}0.4 \mathrm{cc} . \\
\text { leveloped. }\end{array}$ & $0.6 \mathrm{cc}$. & $0.8 \mathrm{cc}$ \\
\hline 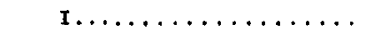 & $9 \cdot 4$ & 6.4 & $6 . I$ & I. 7 & 0.0 & 0.0 \\
\hline $2 \ldots \ldots \ldots \ldots \ldots$ & I5.8 & 12.2 & 10.5 & 2.6 & trace & 0.0 \\
\hline $3 \ldots \ldots \ldots \ldots \ldots$ & 20.2 & 16.3 & $14 \cdot I$ & 3.8 & 0.4 & 0.0 \\
\hline $4 \ldots \ldots \ldots \ldots$ & $23 \cdot 5$ & $19 \cdot 5$ & I6.8 & $4 \cdot 4$ & 0.7 & 0.0 \\
\hline $5 \ldots \ldots \ldots \ldots \ldots$ & $25 \cdot 7$ & 21.8 & 18.8 & $4 \cdot 9$ & 0.9 & 0.0 \\
\hline $6 \ldots \ldots \ldots \ldots \ldots$ & $27 \cdot 4$ & 23.8 & $20 \cdot 3$ & $5 \cdot I$ & I. I & 0.0 \\
\hline $7 \ldots \ldots \ldots \ldots \ldots$ & 28.7 & 25.0 & $2 I \cdot 5$ & $5 \cdot 3$ & I. 2 & trace \\
\hline $8 \ldots \ldots \ldots \ldots \ldots$ & 29.6 & 26.0 & $22 \cdot 5$ & $5 \cdot 5$ & $1 \cdot 3$ & trace \\
\hline $9 \ldots \ldots \ldots \ldots \ldots$ & 30.4 & 26.8 & 23.2 & 5.6 & I. 4 & trace \\
\hline $10 \ldots \ldots \ldots \ldots \ldots$ & $3 \mathrm{I} \cdot 2$ & 27.6 & 23.8 & 5.6 & 1.4 & trace \\
\hline \multicolumn{7}{|c|}{ Effect of Neutralization of Normal $\mathrm{NaOH}$ after I5-Minute Contact. } \\
\hline I $\ldots \ldots \ldots \ldots \ldots$ & 9.4 & 5.6 & $5 \cdot 4$ & 0.7 & 0.0 & 0.0 \\
\hline $2 \ldots \ldots \ldots \ldots \ldots$ & I5.8 & 10.6 & 10.6 & I.9 & trace & 0.0 \\
\hline $3 \ldots \ldots \ldots \ldots \ldots$ & 20.2 & $14 \cdot 4$ & $14 \cdot 3$ & $2 \cdot 4$ & 0.2 & 0.0 \\
\hline $4 \ldots \ldots \ldots \ldots \ldots$ & $23 \cdot 5$ & $17 \cdot 5$ & 16.9 & $2 \cdot 7$ & 0.4 & 0.0 \\
\hline $5 \ldots \ldots \ldots \ldots \ldots$ & $25 \cdot 7$ & I9. 8 & $17 \cdot 3$ & 2.9 & 0.5 & 0.0 \\
\hline $6 \ldots \ldots \ldots \ldots \ldots \ldots$ & 27.4 & 21.6 & 18.4 & 3.2 & 0.7 & 0.0 \\
\hline $7 \ldots \ldots \ldots \ldots \ldots$ & 28.7 & 23.0 & 19.3 & 3.2 & 0.8 & 0.0 \\
\hline $8 \ldots \ldots \ldots \ldots \ldots \ldots$ & 29.6 & $24, I$ & 19.9 & $3 \cdot 3$ & 0.8 & 0.0 \\
\hline $9 \ldots \ldots \ldots \ldots \ldots$ & 30.4 & 25.0 & 20.3 & $3 \cdot 4$ & 0.9 & 0.0 \\
\hline 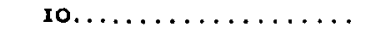 & 31.2 & $25 \cdot 7$ & 20.8 & $3 \cdot 5$ & 0.9 & 0.0 \\
\hline
\end{tabular}


TABLE XI.

Effect of I5-Minute Contact with Normal $\mathrm{NH}_{4} \mathrm{OH}$.

\begin{tabular}{|c|c|c|c|c|c|c|}
\hline \multirow{2}{*}{$\begin{array}{l}\text { Time aiter } \\
\text { adding } \\
\mathrm{H}_{2} \mathrm{O}_{2} \\
\text { (Minutes.) }\end{array}$} & \multicolumn{6}{|c|}{ Normal $\mathrm{NH}_{4} \mathrm{OH}$ added. } \\
\hline & $0 \mathrm{cc}$ & $0.2 \mathrm{cc}$. & $\begin{array}{l}0.4 \mathrm{ce} \\
\text { Oxygen }\end{array}$ & $\begin{array}{l}0.8 \mathrm{ec} \\
\text { eloped. }\end{array}$ & $5 \mathrm{se}$ & $10 \mathrm{cc}$. \\
\hline I. $\ldots \ldots \ldots \ldots \ldots$ & 8.8 & 6.3 & 6.4 & $6 . \mathrm{I}$ & $4 \cdot 9$ & 3.2 \\
\hline $2 \ldots \ldots \ldots \ldots \ldots$ & $14 \cdot 4$ & $12 \cdot 3$ & $I 2.6$ & I 2.3 & 9.5 & $7 \cdot 4$ \\
\hline $3 \ldots \ldots \ldots \ldots$ & 18.7 & 16.4 & I 7.2 & 17.1 & I $3 \cdot 3$ & II 6 \\
\hline$+\ldots \ldots \ldots \ldots$ & 21.6 & 20.0 & 20.6 & 20.8 & 16.1 & I5. 0 \\
\hline $5 \ldots \ldots \ldots \ldots$ & $24 \cdot 3$ & 22.5 & 23.2 & 23.2 & 18.4 & I7. 8 \\
\hline $6 \ldots \ldots \ldots \ldots \ldots$ & 26.3 & $24 \cdot 5$ & $25 \cdot 3$ & 25.0 & 20.1 & $20 . I$ \\
\hline $7 \ldots \ldots \ldots \ldots$ & 27.8 & 26.2 & 26.9 & 26.4 & 21.6 & 22.0 \\
\hline $8 \ldots \ldots \ldots \ldots \ldots$ & $29 \cdot 4$ & $27 \cdot 3$ & 28.1 & 27.6 & 22.8 & $23 \cdot 4$ \\
\hline $9 \ldots \ldots \ldots \ldots$ & 30.7 & 28.6 & $29 \cdot I$ & 28.5 & 24.0 & $24 \cdot 7$ \\
\hline $10 \ldots \ldots \ldots \ldots \ldots$ & $3 I .4$ & 31.1 & 30.0 & $29 \cdot 3$ & 24.8 & $25 \cdot 9$ \\
\hline \multicolumn{7}{|c|}{ Effect of Neutralization of $\mathrm{NH}_{4} \mathrm{OH}$ after 15 -Minute Contact. } \\
\hline$x \ldots \ldots \ldots \ldots \ldots$ & 8.8 & $4 \cdot I$ & $2 \cdot 4$ & $1: 0$ & 0.0 & trace \\
\hline $2 \ldots \ldots \ldots \ldots \ldots$ & $14 \cdot 4$ & 8.5 & 5.9 & $3 \cdot 2$ & trace & trace \\
\hline $3 \ldots \ldots \ldots \ldots \ldots$ & 18.7 & $12 \cdot 3$ & 8.9 & $5 \cdot 2$ & 0.2 & trace \\
\hline $4 \ldots \ldots \ldots \ldots \ldots$ & 21,6 & $15 \cdot 4$ & II. 3 & 6.6 & 0.2 & trace \\
\hline $5 \ldots \ldots \ldots \ldots \ldots$ & $24 \cdot 3$ & 18.0 & 13.9 & 7.9 & 0.2 & trace \\
\hline $6 \ldots \ldots \ldots \ldots \ldots$ & 26.3 & 20.1 & I5.8 & 9.0 & 0.2 & trace \\
\hline $7 \ldots \ldots \ldots \ldots$ & 27.8 & 21.7 & $17 \cdot 4$ & IO.I & 0.2 & trace \\
\hline $8 \ldots \ldots \ldots \ldots$ & 29.4 & 23.0 & 18.5 & 10.9 & 0.3 & trace \\
\hline $9 \ldots \ldots \ldots \ldots$ & 30.7 & 24.1 & 19.9 & 11.6 & 0.3 & trace \\
\hline $10 \ldots \ldots \ldots \ldots \ldots$ & $3 I \cdot 4$ & $25 \cdot I$ & 20.3 & 12.2 & 0.3 & trace \\
\hline
\end{tabular}

TABLE XII.

Fffect of I5-Minute Contact with Normal $\mathrm{Na}_{2} \mathrm{CO}_{3}$.

\begin{tabular}{|c|c|c|c|c|c|}
\hline \multirow{2}{*}{$\begin{array}{l}\text { Time after } \\
\text { adding } \\
\mathrm{H}_{2} \mathrm{O}_{2} . \\
\text { (Minutes.) }\end{array}$} & \multicolumn{5}{|c|}{ Normal $\mathrm{Na}_{2} \mathrm{CO}_{3}$ added. } \\
\hline & $0 \mathrm{cc}$ & $0.1 \mathrm{cc}$ & \multicolumn{2}{|c|}{$\underset{\text { Oxygen developed. }}{0.2 \mathrm{cc} .} \quad 0.4 \mathrm{cc}$} & $0.8 \mathrm{cc}$. \\
\hline $\quad \ldots \ldots \ldots \ldots \ldots$ & 9.0 & $7 \cdot 2$ & $7 \cdot 7$ & 6.4 & 6.4 \\
\hline $2 \ldots \ldots \ldots \ldots \ldots$ & $15 \cdot 7$ & 13.1 & 13.6 & I $2 \cdot 3$ & 12.3 \\
\hline $3 \ldots \ldots \ldots \ldots$ & 20.0 & I $7 \cdot 2$ & 17.8 & 16.5 & 16.3 \\
\hline $4 \ldots \ldots \ldots \ldots \ldots$ & $23 \cdot 3$ & 20.4 & 20.8 & I9.7 & $19 \cdot 5$ \\
\hline $5 \ldots \ldots \ldots \ldots \ldots$ & 26.2 & 23.0 & 23.0 & $22 \cdot 3$ & $2 \mathrm{I}, 7$ \\
\hline $6 \ldots \ldots \ldots \ldots \ldots$ & 28.3 & 25.0 & $24 \cdot 7$ & 24.2 & 23.6 \\
\hline $7 \ldots \ldots \ldots \ldots$ & 30.2 & 26.5 & 26.3 & $25 \cdot 7$ & 25.0 \\
\hline $8 \ldots \ldots \ldots \ldots \ldots \ldots$ & 31.6 & 27.8 & $27 \cdot 5$ & 26.9 & 26.3 \\
\hline $9 \ldots \ldots \ldots \ldots \ldots$ & $32 \cdot 7$ & 28.8 & 28.4 & 28.0 & 27.2 \\
\hline ro................. & 33.6 & $29 \cdot 7$ & $29 \cdot 4$ & 28.8 & 28. I \\
\hline \multicolumn{6}{|c|}{ Effect of Neutralization of Normal $\mathrm{Na}_{2} \mathrm{CO}_{3}$ after I5-Minute Contact. } \\
\hline I $\ldots \ldots \ldots \ldots \ldots$ & 9.0 & 6.6 & $5 \cdot 7$ & $4 \cdot 5$ & $4 \cdot 5$ \\
\hline $2 \ldots \ldots \ldots \ldots \ldots \ldots$ & $5 \cdot 7$ & 12.7 & 10.6 & 8.9 & 8. I \\
\hline $3 \ldots \ldots \ldots \ldots \ldots$ & 20.0 & I5.8 & $14 \cdot 3$ & 12.4 & 10.6 \\
\hline $4 \ldots \ldots \ldots \ldots \ldots$ & $23 \cdot 3$ & 18.9 & $17 \cdot 3$ & 14.9 & I2.7 \\
\hline $5 \ldots \ldots \ldots \ldots \ldots$ & 26.2 & $21 \cdot 3$ & $19 \cdot 7$ & 17.0 & 14.2 \\
\hline $6 \ldots \ldots \ldots \ldots \ldots$ & 28.3 & $23 \cdot 3$ & 21.6 & 18.6 & 15.6 \\
\hline $7 \ldots \ldots \ldots \ldots$ & 30.2 & 25.0 & $2.3 \cdot 1$ & 20.0 & I6. 6 \\
\hline $8 \ldots \ldots \ldots \ldots \ldots \ldots$ & 31.6 & 26.2 & $24 \cdot 4$ & 21.0 & 17.4 \\
\hline $9 \ldots \ldots \ldots \ldots$ & 32.7 & $27 \cdot 3$ & $25 \cdot 5$ & 21.9 & 18.1 \\
\hline 10, $\ldots \ldots \ldots \ldots \ldots \ldots \ldots$ & 33.6 & 28.1 & 26.2 & 22.6 & I8.6 \\
\hline
\end{tabular}


acids was not studied but it is quite probable that the same observations would hold true, as all show a much less inhibiting action toward catalase.

Having shown the effect of some of the more important inorganic and organic acids toward the catalase of taka-diastase, we next tried the effect of alkalies. The methods used in this work were the same as those employed in the previous experiments. The effect of various concentrations of alkalies on the enzyme solution was studied, and a parallel experiment was also performed with each alkali included in this series to show the effect of exactly neutralizing the alkali with standard hydrochloric acid after fifteen-minute contact of enzyme with alkali. The results are expressed in the preceding tables.

In addition to the results given in the preceding tables for sodium carbonate, tests were made with much stronger concentrations of the alkali. The maximum concentration used was $5 \mathrm{cc}$. of twice normal sodium carbonate. In no instance was it possible to effect a lowering of the enzyme activity beyond the point given in the table for $0.8 \mathrm{cc}$. concentration. Neutralization of the alkali with acid showed a decided decrease in activity. Additional concentrations of alkali, besides those given in the table, were not tried with potassium carbonate and acid sodium carbonate.

TABLE XIII.

Effect of ${ }_{15}$-Minute Contact with Normal $\mathrm{K}_{2} \mathrm{CO}_{3}$.

\begin{tabular}{|c|c|c|c|c|c|}
\hline \multirow{3}{*}{ 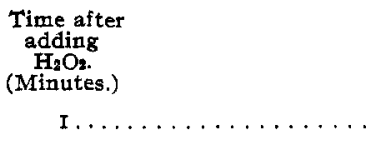 } & \multicolumn{5}{|c|}{ Normal $\mathrm{K}_{2} \mathrm{CO}_{3}$ added. } \\
\hline & \multirow{2}{*}{$\begin{array}{l}0 \mathrm{cc} . \\
8.3\end{array}$} & \multirow{2}{*}{$\begin{array}{l}0.1 \mathrm{cc} . \\
7 \cdot 7\end{array}$} & \multicolumn{2}{|c|}{$\begin{array}{l}0.2 \mathrm{cc} \\
\text { Oxygen developed. }\end{array}$} & $0.8 \mathrm{cc}$ \\
\hline & & & 7.0 & 6.6 & 6.3 \\
\hline $2 \ldots \ldots \ldots \ldots \ldots$ & I5.O & I $4 . I$ & 13.2 & 12.6 & I 2.6 \\
\hline $3 \ldots \ldots \ldots \ldots \ldots \ldots$ & I9. 4 & $19 \cdot 3$ & 18.5 & 17,2 & 17.0 \\
\hline $4 \ldots \ldots \ldots \ldots \ldots$ & $23 \cdot 2$ & 22.8 & 22.1 & 20.6 & 20.2 \\
\hline $5 \ldots \ldots \ldots \ldots \ldots \ldots$ & 26.2 & $25 \cdot 9$ & 24.6 & 23.6 & 23.6 \\
\hline $6 \ldots \ldots \ldots \ldots \ldots$ & 28.3 & $27 \cdot 4$ & 26.3 & 25.6 & $24 \cdot 3$ \\
\hline $7 \ldots \ldots \ldots \ldots \ldots \ldots$ & 30.0 & 28.7 & $27 \cdot 9$ & $27 \cdot 2$ & $25 \cdot 7$ \\
\hline $8 \ldots \ldots \ldots \ldots \ldots \ldots$ & $3 I \cdot 3$ & 29.8 & 29.0 & 28.0 & 27.0 \\
\hline $9 \ldots \ldots \ldots \ldots \ldots \ldots$ & $32 \cdot 3$ & 30.6 & 29.6 & 28.9 & 27.9 \\
\hline го............. & 33.2 & $3 I \cdot 3$ & 30.7 & 29.8 & 28.7 \\
\hline
\end{tabular}

Effect of Neutralization of Normal $\mathrm{K}_{2} \mathrm{CO}_{3}$ after I 5-Minute Contact.

\begin{tabular}{|c|c|c|c|c|c|}
\hline $\mathbf{1} \ldots \ldots \ldots \ldots \ldots \ldots$ & 8.3 & 6.0 & $5 \cdot 7$ & 5.8 & $4 \cdot x$ \\
\hline $2 \ldots \ldots \ldots \ldots \ldots \ldots, \ldots$ & 15.0 & II. 8 & 10.6 & 10.0 & $7 \cdot 2$ \\
\hline $3 \ldots \ldots \ldots \ldots \ldots \ldots$ & 19.4 & 16.4 & I5.0 & I 3.4 & $9 \cdot 7$ \\
\hline $4 \ldots \ldots \ldots \ldots \ldots \ldots$ & 23.2 & 19.9 & 18.2 & 16.2 & II.7 \\
\hline $5 \ldots \ldots \ldots \ldots \ldots \ldots$ & 26.2 & 22.7 & 20.6 & 18.5 & 13.2 \\
\hline $6 \ldots \ldots \ldots \ldots \ldots \ldots$ & 28.3 & $24 \cdot 7$ & $22 \cdot 7$ & 20.2 & $I_{4}$. \\
\hline $7 \ldots \ldots \ldots \ldots \ldots \ldots$ & 30.0 & 26.3 & $24 \cdot I$ & 21.7 & I 5.2 \\
\hline $8 \ldots \ldots \ldots \ldots \ldots \ldots \ldots$ & $3 \mathrm{I} \cdot 3$ & 27.7 & 25.5 & 22.9 & I5. \\
\hline $9 \ldots \ldots \ldots \ldots \ldots \ldots$ & $32 \cdot 3$ & 28.8 & 26.6 & 23.9 & I6. \\
\hline $0 \ldots \ldots \ldots \ldots \ldots$ & 33.2 & 29.7 & 27.4 & 24.6 & \\
\hline
\end{tabular}


TABLE XIV.

Effect of ${ }_{15}$-Minute Contact with Normal $\mathrm{NaHCO}_{3}$.

\begin{tabular}{|c|c|c|c|c|c|}
\hline \multirow{2}{*}{$\begin{array}{l}\text { Time after } \\
\text { adding } \\
\mathrm{H}_{2} \mathrm{O}_{2} \text {. } \\
\text { (Minutes.) }\end{array}$} & \multicolumn{5}{|c|}{ Normal $\mathrm{NaHCO}_{3}$ added. } \\
\hline & $0 \mathrm{cc}$ & $0.1 \mathrm{ce}$ & $\begin{array}{c}0.2 \mathrm{cc} . \\
\text { Oxygen developer }\end{array}$ & $0.4 \mathrm{cc}$ & $0.8 \mathrm{ce}$ \\
\hline$\llbracket \ldots \ldots \ldots \ldots \ldots$ & 7.2 & 6.3 & 6.1 & 6.1 & 5.6 \\
\hline $2 \ldots \ldots \ldots \ldots \ldots \ldots$ & $13 \cdot 4$ & 11.9 & $1 \mathrm{I} \cdot 7$ & II. 7 & II. 2 \\
\hline $3 \ldots \ldots \ldots \ldots \ldots \ldots$ & 18.2 & 16.6 & 16.2 & 16.1 & $15 \cdot 5$ \\
\hline $4 \ldots \ldots \ldots \ldots \ldots$ & 2 I. 9 & 20.2 & 19.6 & I9.6 & 18.9 \\
\hline $5 \ldots \ldots \ldots \ldots \ldots \ldots$ & 24.8 & $23 \cdot 3$ & 22.5 & $22 \cdot 3$ & $2 \mathrm{I} .7$ \\
\hline $6 \ldots \ldots \ldots \ldots \ldots \ldots$ & $27 \cdot 1$ & $25 \cdot 4$ & $24 \cdot 7$ & 24.6 & 23.9 \\
\hline $7 \ldots \ldots \ldots \ldots \ldots \ldots$ & 29.0 & $27 \cdot 3$ & 26.6 & 26,4 & $25 \cdot 7$ \\
\hline $8 \ldots \ldots \ldots \ldots \ldots \ldots$ & 30.5 & 28.7 & 28.0 & $27 \cdot 9$ & 27.2 \\
\hline $9 \ldots \ldots \ldots \ldots \ldots \ldots$ & 31.5 & 29.8 & $29 . \mathrm{I}$ & 28.9 & 28.4 \\
\hline ro $\ldots \ldots \ldots \ldots \ldots \ldots$ & 32.6 & 30.7 & 30.1 & 29.9 & $29 \cdot 3$ \\
\hline
\end{tabular}

Effect of Neutralization of Normal $\mathrm{NaHCO}_{3}$ after I $_{5}$-Minute Contact.

\begin{tabular}{|c|c|c|c|c|c|}
\hline$\mp \ldots \ldots \ldots \ldots \ldots \ldots$ & 7.2 & $5 \cdot 4$ & 4.8 & $4 \cdot 3$ & I. 7 \\
\hline $2 \ldots \ldots \ldots \ldots \ldots \ldots$ & 13.4 & II.I & 10.0 & 8.9 & 4.2 \\
\hline $3, \ldots \ldots \ldots \ldots \ldots$ & 18.2 & 15.6 & I $4 . I$ & $12 \cdot 3$ & 6.7 \\
\hline $4 \ldots \ldots \ldots \ldots \ldots \ldots$ & 21.9 & 19.2 & 17.4 & $x_{5} \cdot 2$ & 8.8 \\
\hline $5 \ldots \ldots \ldots \ldots \ldots \ldots \ldots$ & 24.8 & 21.9 & 20.1 & 17.6 & 10.5 \\
\hline $6 \ldots \ldots \ldots \ldots \ldots \ldots$ & 27.1 & $24 . I$ & 22.0 & 19.5 & II.9 \\
\hline $7 \ldots \ldots \ldots \ldots \ldots \ldots$ & 29.0 & $25 \cdot 7$ & $23 \cdot 7$ & 21.0 & 13.0 \\
\hline $8 \ldots \ldots \ldots \ldots \ldots \ldots$ & 30.5 & $27 \cdot 1$ & $25 \cdot I$ & 22.1 & 13.8 \\
\hline $9 \ldots \ldots \ldots \ldots \ldots \ldots$ & $31 \cdot 5$ & 28.2 & 26.2 & 23.3 & 14.5 \\
\hline$\ldots \ldots \ldots \ldots \ldots \ldots$ & 32.6 & 29.1 & 27.2 & 24.1 & 15.1 \\
\hline
\end{tabular}

The preceding work shows the relative inhibiting effect of bases when in contact with the enzyme solution for fifteen minutes. The following tables show the inhibiting effect of 0.4 cc. normal $\mathrm{NaOH}$ and $\mathrm{KOH}$ on the taka-diastase solution when allowed to remain in contact for different periods of time:

TABLE XV.

Effect of $0.4 \mathrm{cc}$. Normal NaOH for Different Periods of Contact.

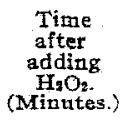

6.8

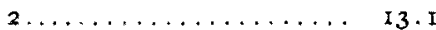

$3 \ldots \ldots \ldots \ldots \ldots \ldots . . \ldots$

$4 \ldots \ldots \ldots \ldots 21.6$

$\$ \ldots \ldots \ldots \ldots \ldots \ldots \ldots$

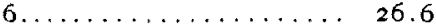

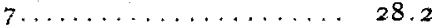

$8 \ldots \ldots \ldots \ldots \ldots \ldots . \ldots \ldots$

9.................. 30.8

I0................ 31.8

\begin{tabular}{rccc}
\multicolumn{4}{c}{$\begin{array}{l}\text { O.4 ce. normal NaOH added. } \\
\text { Time of contact in minutes. }\end{array}$} \\
\hline 0. & $\begin{array}{c}\text { 15. } \\
\text { Oxygen developed. }\end{array}$ \\
4.5 & 2.0 & 1.9 & 0.7 \\
9.2 & 3.9 & 3.8 & 1.7 \\
10.5 & 5.6 & 5.2 & 2.4 \\
16.2 & 6.8 & 6.4 & 3.0 \\
18.5 & 7.9 & 7.3 & 3.4 \\
20.1 & 8.7 & 8.0 & 3.8 \\
21.4 & 9.3 & 8.5 & 4.0 \\
22.4 & 9.8 & 8.9 & 4.2 \\
23.1 & 10.1 & 9.2 & 4.4 \\
23.8 & I0.4 & 9.4 & 4.5
\end{tabular}

The action of bases toward catalase is somewhat similar to the action of acids. Sodium and potassium hydroxide are nearly equal in their effect and they show a much stronger reduction of the activity than the remain- 
TABLE XVI.

Effect of 0.4 cc. Normal KOH for Different Periods of Contact.

\begin{tabular}{|c|c|c|c|c|c|}
\hline \multirow{2}{*}{$\begin{array}{l}\text { Time } \\
\text { after } \\
\text { adding } \\
\mathrm{H}_{2} \mathrm{O}_{2} \\
\text { (Minutes.) }\end{array}$} & \multirow[b]{2}{*}{ Control. } & \multicolumn{4}{|c|}{$\begin{array}{l}0.4 \mathrm{cc} \text {. normal } \mathrm{KOH} \text { added. } \\
\text { Time of contact in minutes. }\end{array}$} \\
\hline & & 0. & \multicolumn{2}{|c|}{$\begin{array}{l}\text { 15. } \\
\text { Oxygen developed. }\end{array}$} & 60. \\
\hline $\mathbf{1} \ldots \ldots \ldots \ldots \ldots \ldots$ & $7 \cdot 9$ & 2.9 & I. 4 & I.O & 0.3 \\
\hline $2 \ldots \ldots \ldots \ldots \ldots \ldots$ & $\mathrm{I} 4 \cdot \mathrm{I}$ & 6.1 & 2.9 & I. 8 & I.O \\
\hline $3 \ldots \ldots \ldots \ldots \ldots \ldots$ & I 8.5 & $9 \cdot 3$ & $4 \cdot I$ & 2.7 & I. 4 \\
\hline $4 \ldots \ldots \ldots \ldots \ldots \ldots$ & 21.8 & I I . 8 & $5 \cdot 2$ & $3 \cdot 9$ & I. 8 \\
\hline $5 \ldots \ldots \ldots \ldots \ldots \ldots$ & $24 \cdot 5$ & I3 $\cdot 7$ & 6.0 & $4 \cdot 1$ & 2.0 \\
\hline $6 \ldots \ldots \ldots \ldots \ldots \ldots$ & 26.7 & I $5 \cdot 3$ & 6.7 & 4.6 & 2.2 \\
\hline $7 \ldots \ldots \ldots \ldots \ldots$ & $29 \cdot 3$ & I 6.4 & $7 \cdot 2$ & $5 \cdot 1$ & $2 \cdot 4$ \\
\hline $8 \ldots \ldots \ldots \ldots \ldots \ldots$ & 29.8 & I 7.6 & $7 \cdot 5$ & $5 \cdot 3$ & $2 \cdot 5$ \\
\hline $9 \ldots \ldots \ldots \ldots \ldots \ldots$ & 30.9 & 18.3 & $7 \cdot 7$ & 5.6 & 2.6 \\
\hline Iо $\ldots \ldots \ldots \ldots \ldots \ldots$ & 31.8 & I 8.8 & 8.0 & $5 \cdot 7$ & 2.7 \\
\hline
\end{tabular}

ing alkalies. Ammonium hydroxide, potassium carbonate, sodium carbonate and sodium bicarbonate show only a slight depressing effect. It is a noticeable fact that neutralization of the alkali by a standard solution of hydrochloric acid increases the retarding effect in every instance.

The duration of contact with sodium and potassium hydroxide has a decided effect upon the enzyme activity. The results show a lowering of enzyme activity when the time of contact is increased.

The results obtained with catalase preparations from different sources, however, are hardly comparable, as it is only reasonable to suppose that the purity of the catalase solutions materially affects the results.

Summary.

I. The inhibiting effect of acids and alkalies upon the catalase of takadiastase follows the order of their ionization constants.

II. After fifteen-minute contact with acid or alkali, the activity of the enzyme cannot be restored by neutralization.

III. The effect of acids was not influenced by the time of contact within the limits of these experiments. The effect of caustic alkalies, on the other hand, was proportional to the period of contact.

In conclusion, the writer takes pleasure in acknowledging his indebtedness to A. W. Dox for his kind suggestions in carrying out this work.

AMEs, IOWA.

[CONTRIBUTION FROM THE HAWAII EXPERIMENT STATION No. 11.$]$

\section{THE ORGANIC NITROGEN OF HAWAIIAN SOILS. I. THE PRODUCTS OF ACID HYDROLYSIS. \\ By W. P. KELLEY. \\ Received November 10, 1913. \\ Introduction.}

The vegetable proteins occurring in different plants constitute the principal source of the soil nitrogen. These undergo biological decompo- 